\title{
LA OFERTA DE TURISMO MÉDICO EN LA CIUDAD DE CARTAGENA
}

\section{RESUMEN}

En esta investigación se analiza la oferta de turismo médico en la ciudad de Cartagena, con el fin de realizar su caracterización y proponer estrategias que permitan superar las barreras que lo obstaculizan. Se aplicó un instrumento de captura de la información dividido en seis capítulos. La información captada fue tabulada y procesada utilizando el software estadístico SPSS versión 19. Se encontró que a pesar de las ventajas que presenta esta actividad en Cartagena, los problemas asociados con la escasez de infraestructura hospitalaria y de profesionales de la salud, sumado a las necesidades de salud locales y bajos niveles de manejo de idioma extranjero, son las principales barreras para su desarrollo; por eso las recomendaciones apuntan a dar soluciones a ello.

Palabras clave: Turismo; Salud; Desarrollo; Servicio; Turismo médico. 


\section{FRANCISCO JOSÉ ARIAS ARAGONÉS* ALEXANDER MAURICIO CARABALLO PAYARES* RODOLFO ENRIQUIE MATOS NAVAS*}

\section{THE SUPPLY OF MEDICAL TOURISM IN CARTAGENA}

\section{ABSTRACT}

This paper analyses and evaluates the medical tourism offer of the Cartagena's city in order to perform the characterization of this and propose strategies to overcome barriers to medical tourism. For the characterization of the supply of medical tourism a tool for capturing the information was used, which was tabulated and processed using the statistical software sPSS. The study found that despite the advantages of this activity in Cartagena, the problem associated with the lack of hospital infrastructure and healthcare, the needs of local health and low levels of handling foreign languages are the main barriers. Therefore, the recommendations point in this direction.

Keywords: Tourism; Health; Development; Services; Medical Tourism.

\section{INTRODUCCIÓN}

La globalización ha afectado todas las actividades económicas directa o indirectamente, dando lugar al surgimiento de nuevas formas de opor- tunidades, procesos y organizaciones de negocios (Vargas, 2005). Los sectores prestadores de servicios de salud no han sido la excepción de esta tendencia, pues muchos países han abierto sus sistemas de salud para una mayor circulación transfronteriza de los pacientes (Smith, Martínez \& Chanda, 2011). Los altos costos de las cirugías y las largas listas de espera, las nuevas tecnologías y habilidades médicas en los países de destino, junto con menores costos de transporte y el marketing en internet han jugado un papel importante (Connell, 2006; Horowitz \& Rosensweig, 2007).

El turismo de salud, o con fines de salud, ha cambiado los parámetros tradicionales asociados a la prestación trasnacional de estos servicios. En otras épocas, los habitantes de países con bajos y medianos ingresos se trasladaban a los países industrializados en busca de atención especializada. En la actualidad, muchos de los países del primer grupo se han hecho famosos por su buena atención médica, tratamiento de enfermedades y rehabilitación, cirugía y otros procesos, ofrecidos a costos mucho más reducidos que aquellos que ofrecen los países de origen de los turistas (Alleyne, 1990; Vargas, 1993).
* Magíster, docente tiempo completo Universidad San Buenaventura. Cartagena, Colombia.Correo-e: farias@usbctg.edu.co.

Magíster, docente tiempo completo Universidad San Buenaventura. Cartagena, Colombia. Correo-e: acaraballo@usbctg.edu.co.

* Magíster, docente tiempo completo Universidad San Buenaventura. Cartagena, Colombia.Correo-e: rmatos@usbctg.edu.co.

Recibido: 14 de octubre de 2014, aceptado: marzo 2 de 2015.

Para citar el artículo: Arias, F.J.; Caraballo A.M.; Matos, R.E. (2015). "La oferta de turismo médico en la ciudad de Cartagena", en Sotavento MBA, n. ${ }^{\circ}$ 25, pp. 10-16. DOI: http://dx.doi.org/10.18601/01233734.n25.02 


\section{FUNDAMENTO TEÓRICO}

Existen dos vertientes de estudio del turismo de salud: la médica y la turística. La primera, se encuentra más asociada con la medicina que con el turismo, dado que considera que la motivación principal del turista es la de acudir a los centros médicos; en este proceso el turista utiliza instalaciones y servicios turísticos, como transporte, alojamiento y restaurantes, entre otros. Sin embargo, la motivación principal no es satisfecha por una instalación o servicio turístico. En esta dirección se encuentran las aportaciones de Borman (2004) y de GarcíaAltes (2005). De manera contraria, la turística se encuentra más vinculada con el turismo que con la medicina, debido a que el turista busca la salud en centros turísticos, como balnearios, centros de talasoterapia, spas, etc. En este sentido, encajan los trabajos de Pollock y Williams (2000), San José Arango (2003) y Sánchez Zapata (2006).

En síntesis, la vertiente médica tiene que ver con la prestación de servicios médicos en instalaciones sanitarias, tales como clínicas y hospitales, y la segunda vertiente tiene que ver con motivaciones turísticas (Lopes, Henn, Alén \& Goncalves, 2011).

El marco conceptual que se emplea para proporcionar una panorámica de la industria del turismo médico a través del funcionamiento del mecanismo de mercado es el propuesto recientemente por Heung, Kucukusta \& Song (2010).

El modelo conceptual consta de dos partes: la oferta y la demanda. Esta última representa los factores que influyen en la elección del destino y las opciones de tratamiento médico, los cuales orientan la decisión del turista médico o paciente extranjero. La oferta aborda básicamente la capacidad que tiene un destino para satisfacer las demandas de estos turistas, mientras que la demanda abarca las necesidades de turismo médico que presentan los turistas.

\section{METODOLOGÍA}

La metodología utilizada combina métodos cuantitativos y cualitativos. El estudio es de carácter exploratorio, porque el turismo médico es una nueva área; por lo tanto, se utilizó el enfoque de teoría fundamentada (Strauss \& Corbin, 1998).

Para realizar la caracterización, se aplicó un instrumento de captura de información a las instituciones (clínicas y/o hospitales) y a los profesionales independientes, identificados como exportadores de servicios de salud por la Cámara de Comercio de Cartagena y agremiados recientemente a través del Núcleo de Turismo de Salud de la ciudad. Luego, se procedió a tabular la información y a procesarla en el software estadístico SPSS versión 19.

\section{RESULTADOS}

La infraestructura hospitalaria es insuficiente para la atención de la población local. Se presenta déficit de camas y salas de cirugía. Las autoridades de salud, como el DADIs (2011), han estimado un faltante de 1.016 camas y una razón de 2 camas por cada 1.000 habitantes, cuando la necesidad es de por lo menos 3 y el estándar internacional de 5,5. También se presenta déficit de atención médica y disminución de la oferta de salud pública, lo cual genera mayor presión sobre el sector privado. En medio de este panorama, el turismo médico es una actividad con un gran potencial de desarrollo, pero que demanda instalaciones médicas y servicios de salud, lo cual genera presiones adicionales sobre un sistema de salud con problemas para atender a la población local. 
Cartagena cuenta con un imponente inventario de atractivos naturales, turísticos e históricos, pero existe poca integración de estos con el turismo médico. La tendencia general en la industria es que los pacientes prefieren obtener tratamiento médico en un ambiente relajante, para lo cual se necesita proporcionar instalaciones y servicios que proveen relajación y recreación, no solo para los pacientes, sino también para los acompañantes (Heung et al., 2010). Esto tiene que ver con el manejo integral del paciente y con la cadena de valor de la industria, que en Cartagena presenta problemas de integración con los servicios de bienestar, transporte, alojamiento y recreación, entre otros proveedores de bienes y servicios.

Para el turismo médico no hay marco regulatorio, lo cual impacta de forma negativa por la proliferación de la informalidad y la ausencia de garantías para los pacientes extranjeros, los cuales corren el riesgo de caer en manos de personas inescrupulosas y en las llamadas "clínicas de garaje". Tampoco existe claridad de las responsabilidades y obligaciones de los participantes en la cadena del turismo médico, en especial a la hora de responder por una mala práctica médica. El paciente extranjero es atendido dentro de la normatividad del sistema de salud colombiano, el cual carece de regulación para los procesos de entrada, salida, protección y seguimiento.

Se encontró que el manejo de idioma extranjero entre los médicos y las enfermeras es del $43 \%$ y $15 \%$, respectivamente. Doce puntos por debajo del promedio nacional en el caso de los médicos y en mejor situación para las enfermeras, dado que a nivel nacional el bilingüismo en esta ocupación es del $8 \%$. Los países más competitivos en esta industria, como Tailandia, exhiben indicadores de bilingüismo entre los médicos del $80 \%$ y $50 \%$ en el personal de enfermería y la India del $100 \%$ en ambas ocupaciones (Ministerio de Comercio, Industria y Turismo, 2009).

Desde el año 2009, el Ministerio de Comercio, Industria y Turismo viene promocionando la actividad del turismo médico a través del Programa de Transformación Productiva (PTP). Recientemente se lanzó el sello "Colombia es Salud, Exportador de Servicios de Salud y Bienestar", con lo cual se pretende destacar el compromiso, innovación y calidad en la prestación de los servicios de salud. Pero la realidad es que Cartagena como destino del turismo médico todavía no muestra un hospital o clínica con una marca icónica, que pueda apoyar la creación y promoción de productos de turismo médico.

El $72 \%$ manifestó apoyo por parte del gobierno: a través del PTP el sector está recibiendo apoyo para la capacitación del personal en idioma extranjero y para la conformación de núcleos empresariales con la ayuda de las cámaras de comercio locales. También se implementó una herramienta de autoevaluación para el sector hotelero interesado en hospedar a estos turistas de la salud.

En Colombia hay escasez de profesionales de la salud; según el Ministerio de la Protección Social (2008), faltan al menos 2.184 médicos. También hay déficit en profesiones como odontología, enfermería, bacteriología, nutrición y dietética para todas las proyecciones de crecimiento del sistema de salud. En Cartagena, el panorama también es de escasez tanto de médicos especialistas como de enfermeras con experiencia y cualificación. Para las clínicas y hospitales es difícil encontrar médicos especialistas y, más difícil, que dominen idioma extranjero.

La oferta de turismo médico se concentra en clínicas y hospitales pertenecientes al sector privado. Estas unidades médicas no son de gran tamaño, pero con la expansión del turismo médico tienen la oportunidad de obtener 
mayores beneficios debido a que el paciente foráneo se encuentra dispuesto a pagar precios más elevados que el paciente local o nacional. Esto ha despertado el interés de inversionistas privados y del gobierno nacional. Sin embargo, estas inversiones podrían verse afectadas por los altos precios y la disponibilidad de la tierra en espacios privilegiados (cerca del mar, aeropuerto y sitios de interés).

\section{CONCLUSIONES Y DISCUSIÓN}

Se recomiendan las siguientes estrategias para levantar las barreras que obstaculizan el desarrollo del turismo médico.

- Infraestructura hospitalaria. Se necesitan más hospitales y clínicas para superar el actual déficit de instalaciones. Los nuevos centros médicos podrían dedicarse por completo a promover ciertas especialidades y productos del turismo médico, como cirugía estética, cardiovascular y bariátrica, las cuales fueron identificadas como las de mayores fortalezas.

- Apoyo del gobierno. Puede apoyar en muchos aspectos, asegurándose de que dicho apoyo no ponga en riesgo la provisión de servicios de salud de la población local. El problema de capacidad del sistema de salud puede ser resuelto mediante el fomento de inversión nacional y extranjera, creándose incentivos y flexibilizando la regulación (deducciones fiscales y apoyo financiero para adquisición de equipos médicos). También puede impulsar alianzas público-privadas para eliminar riesgos financieros, técnicos y operativos de las nuevas inversiones y proyectos de turismo médico. Adicionalmente, las nuevas regulaciones deben proteger al sistema de salud público, puesto que el desarrollo del turismo médico podría desencadenar una desviación de recursos y profesionales del sistema público hacia el sistema privado, situación que hasta ahora no se ha tenido en cuenta en la agenda oficial de promoción del sector. Se sugiere que una parte de los ingresos del turismo médico se inviertan en el fortalecimiento del sistema de salud público local o que se cree algún instrumento de contraprestación que garantice la armonía entre las necesidades de salud locales y las de los foráneos.

- Cualificación y disponibilidad del recurso humano. Este se encuentra por debajo del promedio nacional en el uso y dominio de idioma extranjero, y lejos del promedio de los grandes competidores. Esto dificulta la comunicación con los pacientes durante el tratamiento y proceso de recuperación, haciendo un daño grave a la percepción de confianza y seguridad en la prestación del servicio médico. Hay escasez de médicos especialistas y enfermeras con experiencia y cualificación. Para remediar lo anterior, es necesario que los empresarios inviertan en la formación y capacitación de los empleados y que vinculen personal con estas competencias.

- Marco regulatorio. Se recomienda regular lo antes posible la actividad del turismo médico a través de la expedición de leyes $y$ decretos que permitan controlar los procesos de entrada, salida, protección y seguimiento de los pacientes extranjeros, de tal forma que se puedan establecer las responsabilidades de cada uno de los participantes, con lo cual se cierra y asegura la cadena productiva del turismo médico (Universidad Sergio Arboleda, 2014).

- Promoción. Hasta el momento no ha surgido una clínica u hospital con una marca icónica que pueda apoyar la creación y 
promoción de productos de turismo médico. En otras ciudades, como Bogotá y Medellín, se viene impulsando el turismo médico a través de importantes clínicas y hospitales, como la Fundación Cardioinfantil y el Hospital Pablo Tobón Uribe. Se aconseja que el sector del turismo médico de Cartagena construya su propia marca con una imagen a partir de la buena reputación que tienen los médicos y la calidad de los servicios que prestan. También es importante la promoción por parte del gobierno a través del Ministerio de Industria, Turismo y Comercio, al incluir este tipo de turismo dentro de la oferta turística de las ciudades colombianas.

- Instalaciones. Se requiere colaboración entre el sector de la salud y el turismo por ejemplo; ante la insuficiencia de camas en los hospitales, los hoteles podrían colaborar para atender a las necesidades de estos turistas y las de sus acompañantes. En este sentido, se podría crear una organización que se encargue de la planificación, organización y seguimiento de estas actividades, además de integrar de mejor manera los servicios médicos con los de bienestar, transporte y recreación, entre otros, de tal forma que se consiga un manejo integral del paciente que fortalezca su experiencia como turista de la salud.

\section{REFERENCIAS}

Alleyne, G. (1990). "Health and tourism in the Caribbean", en Bulletin of Paho.

Borman, E. (2004) "Health tourism", en BMJ Medical Publication 328: 60-61. Disponible en: http://www.bmj.com.

Connell, J. (2006). "Medical tourism: sea, sun, sand and ... surgery", en Tourism Management 27 (1093-1100).
DAdis (2011). Perfil Epidemiológico de Cartagena. Programa de Vigilancia en Salud Pública. Disponible en http://www.minsalud.gov.co/sites/rid/ Lists/BibliotecaDigital/RIDE/VS/ED/PSP/Analisisde-Situacion-Salud-Cartagena-2011.pdf.

García-Altes, M. (2005). The development of health tourism services.

Heung, V. C. S, Kucukusta, D. \& Song, H. (2010). "A conceptual model of medical tourism: implications for future research", en Journal of Travel and Tourism Marketing, 27(3), 236-251.

Horowitz, Michael D.; Jeffrey A. Rosensweig \& Christopher A. Jones (2007). "Medical tourism: globalization of the healthcare marketpla-

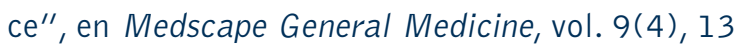
November.

Lopez, P.; Henn, M.; Alén, M. \& Goncalves, J. (2011). "El turismo de salud y el uso terapéutico del agua", en Estudios y perspectivas en turismo. Vol 20.

Ministerio de Comercio, Industria y Turismo (2009). Desarrollando sectores de clase mundial en Colombia. Disponible en http://econotur.files. wordpress.com/2013/02/ptp-20090709-resumenejecutivo-turismo-de-salud.pdf.

Ministerio de la Protección Social (2008). Recursos Humanos de la Salud en Colombia: Balance, Competencias y Prospectiva. Disponible en http://salud.univalle.edu.co/pdf/plan_desarrollo/ recursos_humanos_de_la_salud_en_colombia_cendex_3_edicion.pdf.

Pollock, A.; Williams, P. (2000) "Health tourism trends: closing the gap between health care and tourism", en Gartner, W (Ed.). Trends in outdoor recreation, leisure and tourism. Cabi Publishing, pp.165-173.

San José Arango, C. (1998) Hidrología médica y terapias complementares. Sevilla: Universidad de Sevilla.

Sánchez Zapata, A. (2006) "Turismo de salud: situación actual y perspectivas de futuro", en Apuntes IX Master en Organización y Dirección Turística: Turismo de Salud. Ourense-Universidad de Vigo. 
Smith, R. Martínez, M. \& Chanda, R. (2011). Medical tourism: A review of literature and analysis of a role for bi-lateral trade.

Strauss, A. \& Corbin, J. (1998). Basics of qualitative research: Techniques and procedures for developing grounded theory (2nd ed.). Thousand Oaks, cA: Sage.

Universidad Sergio Arboleda (2014). Estudio para definir y caracterizar la informalidad en 8 sectores seleccionados del Programa de Trans- formación Productiva. Sector Turismo de Salud y Bienestar. Disponible en http://www.ptp.com.co/ documentos/Entregable \%202B\%20Turismo\%20 de $\% 20$ Salud $\% 20 y \% 20$ Bienestar.pdf.

Vargas, J. (2005). Cross border trade in Health Care Services between Tijuana, Mexico and San Diego.

Vargas, M. (1993). Turismo y salud, sus relaciones e importancia. Informe de estudio en la región de las Américas. 
